CRYSTALLOGRAPHIC COMMUNICATIONS

ISSN 2056-9890

Received 2 October 2019

Accepted 12 November 2019

Edited by W. T. A. Harrison, University of Aberdeen, Scotland

Keywords: tetracyanoplatinate; crown ether; platinum; potassium; crystal structure.

CCDC reference: 1965195

Supporting information: this article has supporting information at journals.iucr.org/e
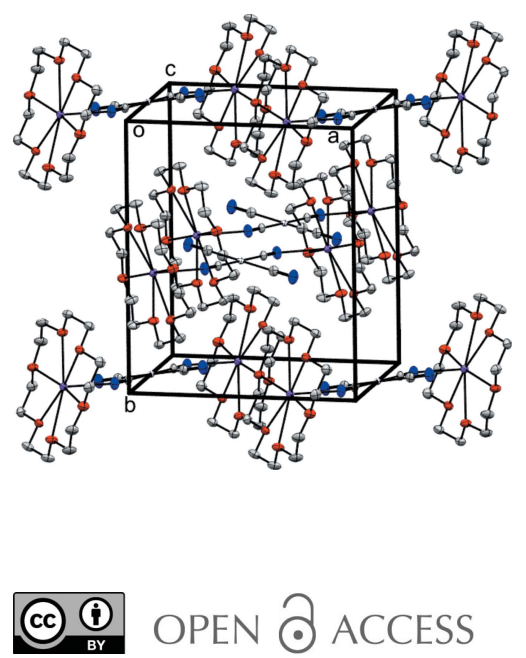

\section{Crystal structure of $[\mathrm{K}(18-\mathrm{crown}-6)]_{2}^{+}\left[\mathrm{Pt}(\mathrm{CN})_{4}\right]^{2-}$}

\author{
Malte Sellin and Moritz Malischewski*
}

Freie Universität Berlin, Institut für Chemie und Biochemie - Anorganische Chemie, Fabeckstrasse 34-36, 14195 Berlin, Germany. *Correspondence e-mail: moritz.malischewski@fu-berlin.de

In the title compound, di- $\mu$-cyanato- $1: 2 \kappa^{2} N: C ; 2: 3 \kappa^{2} C: N$-dicyanato- $2 \kappa^{2} C$-bis $(1,4,-$ 7,10,13,16-hexaoxacyclooctadecane)- $1 \kappa^{6} O ; 3 \kappa^{6} O-1,3$-dipotassium(I)-2-platinum(II), $\left[\mathrm{K}_{2} \mathrm{Pt}(\mathrm{CN})_{4}\left(\mathrm{C}_{12} \mathrm{H}_{24} \mathrm{O}_{6}\right)_{2}\right]$ or $[\mathrm{K}(18 \text {-crown- } 6)]_{2} \cdot\left[\mathrm{Pt}(\mathrm{CN})_{4}\right]$, two transorientated cyano groups of the square-planar $\left[\mathrm{Pt}(\mathrm{CN})_{4}\right]^{2-}$ dianion $(\mathrm{Pt}$ site symmetry $\overline{1}$ ) bind to one potassium ion each, which are additionally coordinated by the six $\mathrm{O}$ atoms of 18 -crown- 6 . No Pt ...Pt interactions occur in the crystal, but very weak Pt...H contacts $(2.79 \AA)$ are observed.

\section{Chemical context}

Polycyanometallates are an important class of inorganic compounds with intriguing properties. As a result of their anionic nature and high nucleophilicity, they have been widely used as metallo-ligands in coordination chemistry. Depending on the geometry of the polycyanometallate, various topologies can be realized (Alexandrov et al., 2015). While photomagnetic effects have been predominantly realized with hexaand octacyanometallates (Ohkoshi et al., 2012), studies on tetracyanoplatinates and their derivatives have focused on the high electrical conductivities of mixed-valent Krogmann's salts $\mathrm{K}_{2}\left[\mathrm{Pt}(\mathrm{CN})_{4}\right] \mathrm{Br}_{0.32} \cdot 2.6 \mathrm{H}_{2} \mathrm{O}$ (Krogmann, 1969), vapochromic sensor materials (e.g. $\mathrm{Zn}\left[\mathrm{Pt}(\mathrm{CN})_{4}\right]$ for ammonia (Varju et al., 2019) and spin-crossover compounds such as $[\mathrm{Fe}($ pyrazine $)]\left[\mathrm{Pt}(\mathrm{CN})_{4}\right] \cdot 2 \mathrm{H}_{2} \mathrm{O}$ (Niel et al., 2001). However, alkali salts of polycyanometallates are in generally watersoluble but suffer from insolubility in organic solvents. A general way to increase the solubility of metals salts in organic solvents is the utilization of crown ethers. For example, even potassium permanganate $\mathrm{KMnO}_{4}$ becomes benzene-soluble by coordination of 18 -crown- 6 to the potassium cation (Doheny \& Ganem, 1980). During our attempts to explore the coordination chemistry of the tetracyanoplatinate dianion $\left[\mathrm{Pt}(\mathrm{CN})_{4}\right]^{2-}$ in organic solvents, we realized that commercially available $\mathrm{K}_{2}\left[\mathrm{Pt}(\mathrm{CN})_{4}\right]$ is insoluble in dichloromethane but dissolves rapidly upon addition of 18-crown-6. The product $\left[\mathrm{K}(18 \text {-crown-6) }]_{2}\left[\mathrm{Pt}(\mathrm{CN})_{4}\right]\right.$, which was already isolated many years ago by a rather complicated procedure (Almeida \& Pidcock, 1981), could now be obtained in crystalline form. In contrast to other tetracyanoplatinate(II) salts with large organic cations $\left[\right.$ e.g. $\mathrm{PPh}_{4}{ }^{+}$(see Nast \& Moerler, 1969) and $\mathrm{NBu}_{4}{ }^{+}$(see Mason \& Gray, 1968)], which are prepared by metathesis reactions in water, this new procedure makes the access to tetracyanoplatinate salts with solubility in organic solvents even more facile. 


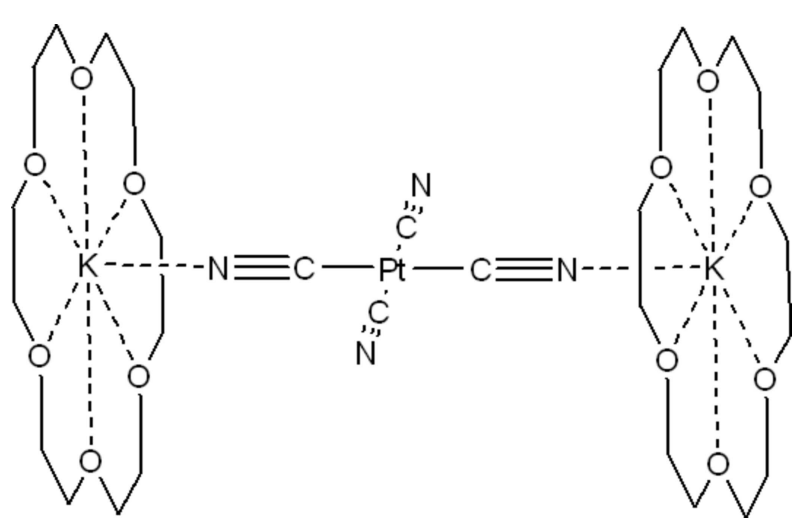

\section{Structural Commentary}

$\left[\mathrm{K}(18 \text {-crown-6) }]_{2}\left[\mathrm{Pt}(\mathrm{CN})_{4}\right]\right.$ (Fig. 1) crystallizes in the monoclinic space group $P 2_{1} / n$. The tetracyanoplatinate moiety displays a square-planar molecular geometry with the platinum atom lying on a crystallographic inversion centre. Two trans-orientated cyano groups coordinate via their terminal nitrogen atoms to the potassium ions in a rather bent fashion $\left[\mathrm{K} 1-\mathrm{N} 1-\mathrm{C} 1=146.76(17)^{\circ}\right]$ while the $\mathrm{Pt}-\mathrm{C}-\mathrm{N}$ bonds are almost linear $\left[\mathrm{Pt} 1-\mathrm{C} 2-\mathrm{N} 2=178.81(18)^{\circ}\right]$. The $\mathrm{Pt}-\mathrm{C}$ and $\mathrm{C}-\mathrm{N}$ bond lengths do not differ significantly between the terminal or bridging cyano ligands $[\mathrm{Pt} 1-\mathrm{C} 2=$ 1.996 (2) $\AA$ versus $\mathrm{Pt} 1-\mathrm{C} 1=1.991(2) \AA$ and $\mathrm{C} 2-\mathrm{N} 2=$ 1.155 (3) $\AA$ versus $\mathrm{C} 1-\mathrm{N} 1=1.154$ (3) $\AA$ ] . The six oxygen atoms of the crown ether coordinate to the potassium ion in a hexagonal-planar fashion. Additionally, one apical position is occupied by a nitrogen atom of a cyano group, although the $\mathrm{K}-\mathrm{N}$ distance is relatively long [2.732 (2) $\AA$ ] . The potassium ion is located $0.295 \AA$ above the the $\mathrm{O}_{6}$ centroid $[\mathrm{K}-\mathrm{O}$ distances $=2.769$ (1)-2.837 (1) $\mathrm{]}]$.

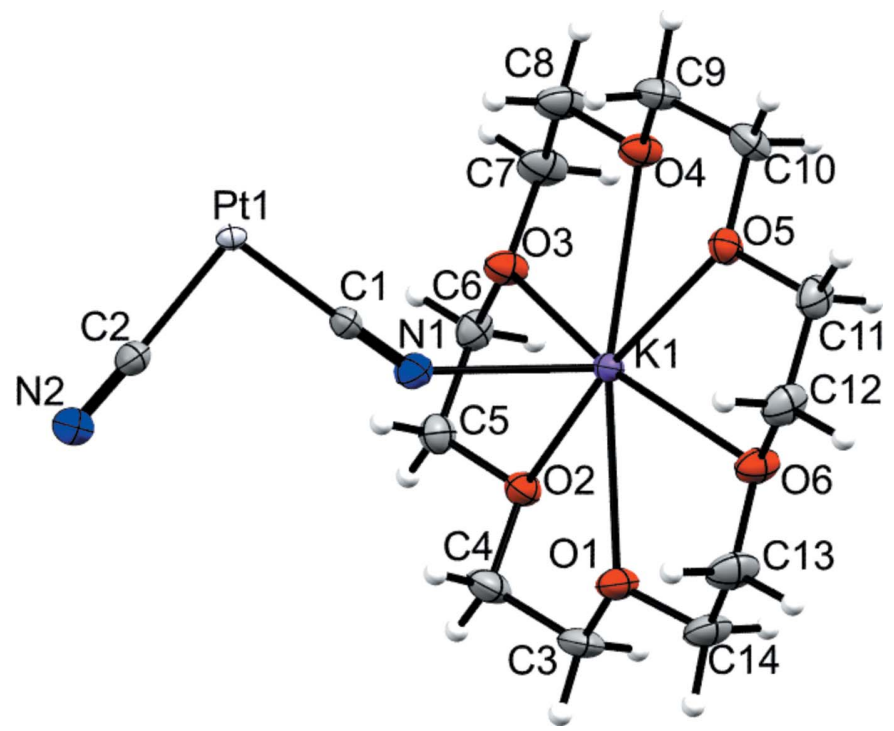

Figure 1

The asymmetric unit of the title compound with displacement ellipsoids shown at the $50 \%$ probability level.
Table 1

Hydrogen-bond geometry $\left(\AA{ }^{\circ}\right)$.

\begin{tabular}{lllll}
\hline$D-\mathrm{H} \cdots A$ & $D-\mathrm{H}$ & $\mathrm{H} \cdots A$ & $D \cdots A$ & $D-\mathrm{H} \cdots A$ \\
\hline $\mathrm{C} 3-\mathrm{H} 3 A \cdots \mathrm{N} 1^{\mathrm{i}}$ & 0.99 & 2.54 & $3.510(3)$ & 165 \\
$\mathrm{C} 9-\mathrm{H} 9 B \cdots \mathrm{N} 2^{\mathrm{ii}}$ & 0.99 & 2.55 & $3.459(3)$ & 152 \\
\hline
\end{tabular}

Symmetry codes: (i) $-x+1,-y+1,-z+1$; (ii) $x+1, y, z$.

\section{Supramolecular features}

A common feature of tetracyanoplatinate salts is the formation of columnar stacks of the planar tetracyanoplatinate anions with Pt...Pt distances in the range of 3.0-3.8 $\AA$, see, for example, Washecheck et al. (1976), Holzapfel et al. (1981), Mühle et al. (2004) and Neuhausen et al. (2011). However, in the crystal structure of the title compound (Fig. 2), no platinophilic interactions are observed. This is in accordance with findings of Stojanovic et al. (2011) who stated that large organic cations can suppress the formation of Pt...Pt contacts. Intermolecular interactions are not very pronounced in this crystal structure. However, the two uncoordinated cyano groups each point towards one neighbouring hydrogen atom in a slightly bent fashion $\left(\mathrm{C}-\mathrm{N} \cdots \mathrm{H}=152^{\circ}\right.$; Table 1$)$ although the $\mathrm{N} \cdots \mathrm{H}$ distance is relatively long $(2.55 \AA)$. Moreover, two hydrogen atoms from two different crown ether molecules form weak contacts to the platinum atom in a linear fashion $\left(\mathrm{H} \cdots \mathrm{Pt} \cdots \mathrm{H}=180^{\circ}\right)$, which results in a distorted axially elongated pseudo-octahedral $\mathrm{PtC}_{4} \mathrm{H}_{2}$ coordination environment for the platinum atom. The Pt $\cdots H$ distances are slightly smaller than the sum of the van der Waals radii $(2.79 \AA)$.

\section{Database survey}

A database survey (CSD version 5.40, update of November 2018; Groom et al., 2016) gave 348 hits for the $\left[\mathrm{Pt}(\mathrm{CN})_{4}\right]$

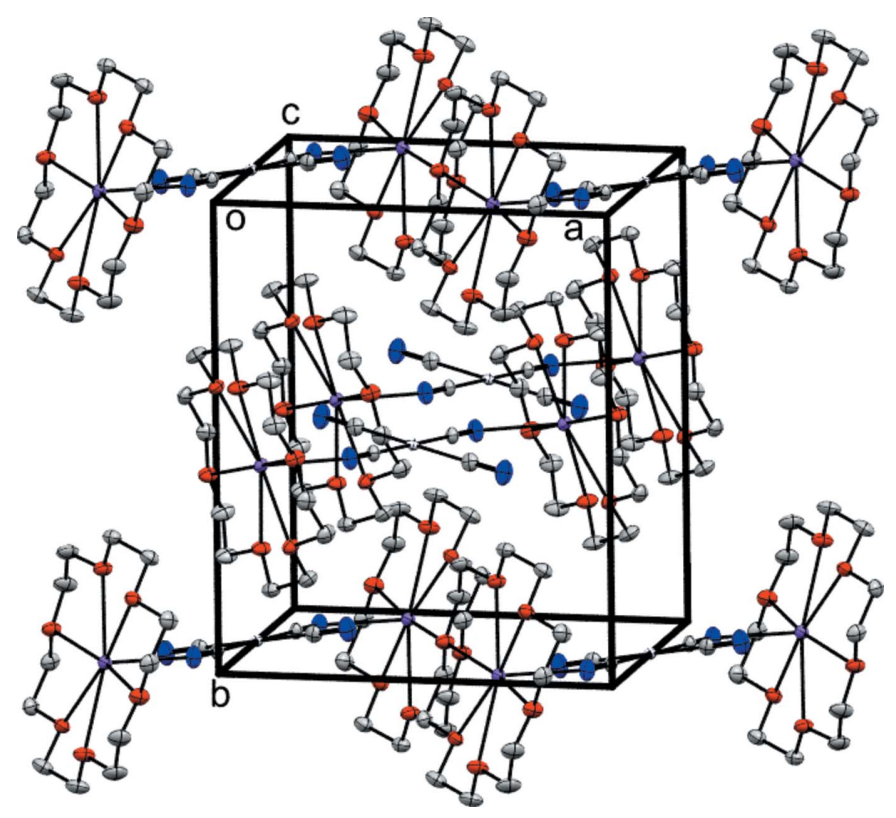

Figure 2

Packing in the unit cell of the title compound. 
moiety and 1562 hits for the [K(18-crown-6)] moiety. While the tetracyanoplatinate moiety binds to many elements from the periodic table, only a few tetracyanoplatinate salts with metal-crown ether counter-cations are known. For example, complexes of $\mathrm{Ba}^{2+}\left[\mathrm{Pt}(\mathrm{CN})_{4}\right]^{2-}$ with 18-crown-6 (Olmstead et al., (2005), dibenzo-18-crown-6 (Olmstead et al., 2016)) and diaza-18-crown-6 (Olmstead et al., 2009). In the first two examples, the $\mathrm{Ba}^{2+}$ cation exhibits a coordination number of 10 whereas only ninefold coordination is observed in the last case. In general, these high coordination numbers result from bridging cyanide ligands and oxygen-containing donor solvents that bind to the $\mathrm{Ba}^{2+}$ cations. In [Tl(18-crown6) $]_{2}\left[\mathrm{Pt}(\mathrm{CN})_{4}\right]$ (Liu et al., 2006), only a sevenfold coordination is observed for the thallium cation. Interestingly, $\mathrm{Tl}^{+}$does not bind to a terminal cyanide group but forms a weak metallophilic contact to $\mathrm{Pt}^{2+}(\mathrm{Tl} \cdots \mathrm{Pt}$ distance $=3.185 \AA)$.

The combination of [K(18-crown-6)] cations with other polycyanometallates is relatively rare. Crystal structures of $\left[\mathrm{K}_{3}(18 \text {-crown-6) })_{3}\left(\mathrm{H}_{2} \mathrm{O}\right)_{4}\right]\left[\mathrm{Cr}(\mathrm{CN})_{6}\right] \cdot 3 \mathrm{H}_{2} \mathrm{O}$ (Zhou et al., 2003), $\left[\mathrm{K}(18 \text {-crown-6) }]_{2}\left[\mathrm{~K}\left(18\right.\right.\right.$-crown-6) $\left.\left(\mathrm{H}_{2} \mathrm{O}\right)_{2}\right]\left[\mathrm{Ru}(\mathrm{CN})_{6}\right] \cdot \mathrm{CH}_{2} \mathrm{Cl}_{2}$ (Vostrikova \& Peresypkina, 2011) and $\left[\mathrm{K}(18 \text {-crown-6) }]_{2}-\right.$ $\left[\mathrm{K}\left(18\right.\right.$-crown-6) $\left.\left(\mathrm{C}_{3} \mathrm{H}_{7} \mathrm{OH}\right)\right]\left[\mathrm{Os}(\mathrm{CN})_{6}\right] \cdot 2 \mathrm{C}_{3} \mathrm{H}_{7} \mathrm{OH} \cdot \mathrm{H}_{2} \mathrm{O}$ (Vostrikova \& Peresypkina, 2011) have been reported in the literature.

\section{Synthesis and crystallization}

Potassium tetracyanoplatinate $(37.7 \mathrm{mg}, 0.1 \mathrm{mmol})$ was suspended in $3 \mathrm{ml}$ of $\mathrm{CH}_{2} \mathrm{Cl}_{2}$. Then, $52.8 \mathrm{mg}(0.2 \mathrm{mmol})$ of 18 crown- 6 were added and the mixture was stirred for several minutes until the solid had completely dissolved. A small part of the solution was placed in a narrow glass tube and layered with diethyl ether. Colourless blocks of the title compound formed overnight. IR(ATR) $\left(\mathrm{cm}^{-1}\right)$ : 2898-2815 [m, $\left.v(\mathrm{CH})\right]$, $2126[s, n(\mathrm{CN})], 1451\left[w, d\left(\mathrm{CH}_{2}\right)\right], 1099[v s, n(\mathrm{CO})] .{ }^{1} \mathrm{H}$ NMR (400 MHz in $\mathrm{CD}_{2} \mathrm{Cl}_{2}$ ): 3.62 ( $s$, crown ether) ppm. ${ }^{13} \mathrm{C}\left({ }^{1} \mathrm{H}\right)$ NMR $\left(101 \mathrm{MHz}\right.$ in $\left.\mathrm{CD}_{2} \mathrm{Cl}_{2}\right): 122.4\left(\mathrm{CN},{ }^{1} J_{\mathrm{Pt}-\mathrm{C}}=1018 \mathrm{~Hz}\right)$, 70.1 (crown) ppm.

\section{Refinement}

Crystal data, data collection and structure refinement details are summarized in Table 2. The $\mathrm{H}$ atoms were placed geometrically with a constrained $\mathrm{C}-\mathrm{H}$ distance of $0.99 \AA$ and refined as riding atoms with $U_{\text {iso }}(\mathrm{H})=1.2 U_{\text {eq }}(\mathrm{C})$.

\section{Funding information}

We acknowledge support by the German Research Foundation and the Open Access Publication Fund of the Freie Universität, Berlin.

\section{References}

Alexandrov, E. V., Virovets, A. V., Blatov, V. A. \& Peresypkina, E. V. (2015). Chem. Rev. 115, 12286-12319.

Almeida, J. F. \& Pidcock, A. (1981). J. Organomet. Chem. 208, 273278.
Table 2

Experimental details.

\begin{tabular}{|c|c|}
\hline \multicolumn{2}{|l|}{ Crystal data } \\
\hline Chemical formula & {$\left[\mathrm{K}_{2} \mathrm{Pt}(\mathrm{CN})_{4}\left(\mathrm{C}_{12} \mathrm{H}_{24} \mathrm{O}_{6}\right)_{2}\right]$} \\
\hline$M_{\mathrm{r}}$ & 905.99 \\
\hline Crystal system, space group & Monoclinic, $P 2_{1} / n$ \\
\hline Temperature $(\mathrm{K})$ & 100 \\
\hline$a, b, c(\AA)$ & $\begin{array}{l}11.7341(10), 13.7280(12) \\
\quad 11.8876(10)\end{array}$ \\
\hline$\beta\left(^{\circ}\right)$ & $94.999(3)$ \\
\hline$V\left(\AA^{3}\right)$ & $1907.6(3)$ \\
\hline$Z$ & 2 \\
\hline Radiation type & Мо $K \alpha$ \\
\hline$\mu\left(\mathrm{mm}^{-1}\right)$ & 3.96 \\
\hline Crystal size (mm) & $0.44 \times 0.44 \times 0.12$ \\
\hline \multicolumn{2}{|l|}{ Data collection } \\
\hline Diffractometer & Bruker APEXII CCD \\
\hline Absorption correction & $\begin{array}{l}\text { Multi-scan (SADABS; Bruker, } \\
\text { 2016) }\end{array}$ \\
\hline$T_{\min }, T_{\max }$ & $0.306,0.564$ \\
\hline $\begin{array}{l}\text { No. of measured, independent and } \\
\text { observed }[I>2 \sigma(I)] \text { reflections }\end{array}$ & $57788,5839,4658$ \\
\hline$R_{\mathrm{int}}$ & 0.047 \\
\hline$(\sin \theta / \lambda)_{\max }\left(\AA^{-1}\right)$ & 0.716 \\
\hline \multicolumn{2}{|l|}{ Refinement } \\
\hline$R\left[F^{2}>2 \sigma\left(F^{2}\right)\right], w R\left(F^{2}\right), S$ & $0.019,0.051,1.05$ \\
\hline No. of reflections & 5839 \\
\hline No. of parameters & 215 \\
\hline $\mathrm{H}$-atom treatment & $\mathrm{H}$-atom parameters constrained \\
\hline$\Delta \rho_{\max }, \Delta \rho_{\min }\left(\mathrm{e} \AA^{-3}\right)$ & $1.25,-1.54$ \\
\hline
\end{tabular}

Computer programs: APEX2 and SAINT (Bruker, 2016), SHELXS (Sheldrick, 2008), SHELXL2018 (Sheldrick, 2015), OLEX2 (Dolomanov et al., 2009) and publCIF (Westrip, 2010).

Bruker (2016). APEX2 and SAINT. Bruker AXS Inc., Madison, Wisconsin, USA.

Doheny, A. J. \& Ganem, B. (1980). J. Chem. Educ. 57, 308.

Dolomanov, O. V., Bourhis, L. J., Gildea, R. J., Howard, J. A. K. \& Puschmann, H. (2009). J. Appl. Cryst. 42, 339-341.

Groom, C. R., Bruno, I. J., Lightfoot, M. P. \& Ward, S. C. (2016). Acta Cryst. B72, 171-179.

Holzapfel, W., Yersin, H. \& Gliemann, G. (1981). Z. Kristallogr. 157, 47-67.

Krogmann, K. (1969). Angew. Chem. Int. Ed. Engl. 8, 35-42.

Liu, F.-H., Chen, W.-Z. \& Wang, D.-Q. (2006). Chin J. Struct. Chem. 25, 677-680.

Mason, W. R. III \& Gray, H. B. (1968). J. Am. Chem. Soc. 90, 57215729.

Mühle, C., Nuss, J., Dinnebier, R. E. \& Jansen, M. (2004). Z. Anorg. Allg. Chem. 630, 1462-1468.

Nast, R. \& Moerler, H.-D. (1969). Chem. Ber. 102, 2050-2056.

Neuhausen, C., Pattison, P. \& Schiltz, M. (2011). CrystEngComm, 13, 430-432.

Niel, V., Martinez-Agudo, J. M., Muñoz, M. C., Gaspar, A. B. \& Real, J. A. (2001). Inorg. Chem. 40, 3838-3839.

Ohkoshi, S.-I. \& Tokoro, H. (2012). Acc. Chem. Res. 45, 1749 1758.

Olmstead, M. M., Beavers, C. M. \& Paw, U. L. (2009). Acta Cryst. E65, m408-m409.

Olmstead, M. M., Lee, M. A. \& Stork, J. R. (2005). Acta Cryst. E61, m1048-m1050.

Sheldrick, G. M. (2008). Acta Cryst. A64, 112-122.

Sheldrick, G. M. (2015). Acta Cryst. C71, 3-8.

Stojanovic, M., Robinson, N. J., Ngo, T. \& Sykora, R. E. (2011). J. Chem. Crystallogr. 41, 1425-1432.

Varju, D. R., Wollschlaeger, S. A. \& Leznoff, D. B. (2019). Chem. Eur. J. 25, 9017-9025. 


\section{research communications}

Vostrikova, K. E. \& Peresypkina, E. V. (2011). Eur. J. Inorg. Chem. pp. 811-815.

Washecheck, D. M., Peterson, S. W., Reis, A. H. Jr \& Williams, J. M. (1976). Inorg. Chem. 15, 74-78.
Westrip, S. P. (2010). J. Appl. Cryst. 43, 920-925.

Zhou, B.-C., Kou, H.-Z., He, Y., Wang, R.-J., Li, Y.-D. \& Wang, H.-G. (2003). Chin. J. Chem. 21, 352-355. 


\section{supporting information}

Acta Cryst. (2019). E75, 1871-1874 [https://doi.org/10.1107/S2056989019015238]

Crystal structure of $[\mathrm{K}(18-\mathrm{crown}-6)]^{+}\left[\mathrm{Pt}(\mathrm{CN})_{4}\right]^{2-}$

\section{Malte Sellin and Moritz Malischewski}

\section{Computing details}

Data collection: APEX2 (Bruker, 2016); cell refinement: SAINT (Bruker, 2016); data reduction: SAINT (Bruker, 2016); program(s) used to solve structure: SHELXS (Sheldrick, 2008); program(s) used to refine structure: SHELXL2018 (Sheldrick, 2015); molecular graphics: OLEX2 (Dolomanov et al., 2009); software used to prepare material for publication: publCIF (Westrip, 2010).

Di- $\mu$-cyanato-1:2 $\kappa^{2} N: C ; 2: 3 \kappa^{2} C: N$-dicyanato- $2 \kappa^{2} C$-bis $\left(1,4,7,10,13,16\right.$-hexaoxacyclooctadecane)-1 $\kappa^{6} O ; 3 \kappa^{6} O-1,3-$ dipotassium(I)-2-platinum(II)

\section{Crystal data}

$\left[\mathrm{K}_{2} \mathrm{Pt}(\mathrm{CN})_{4}\left(\mathrm{C}_{12} \mathrm{H}_{24} \mathrm{O}_{6}\right)_{2}\right]$

$M_{r}=905.99$

Monoclinic, $P 2_{1} / n$

$a=11.7341(10) \AA$

$b=13.7280(12) \AA$

$c=11.8876(10) \AA$

$\beta=94.999(3)^{\circ}$

$V=1907.6(3) \AA^{3}$

$Z=2$

\section{Data collection}

\section{Bruker APEXII CCD}

diffractometer

$\varphi$ and $\omega$ scans

Absorption correction: multi-scan

(SADABS; Bruker, 2016)

$T_{\min }=0.306, T_{\max }=0.564$

57788 measured reflections

\section{Refinement}

Refinement on $F^{2}$

Least-squares matrix: full

$R\left[F^{2}>2 \sigma\left(F^{2}\right)\right]=0.019$

$w R\left(F^{2}\right)=0.051$

$S=1.05$

5839 reflections

215 parameters

0 restraints

Primary atom site location: structure-invariant direct methods
$F(000)=912$

$D_{\mathrm{x}}=1.577 \mathrm{Mg} \mathrm{m}^{-3}$

Mo $K \alpha$ radiation, $\lambda=0.71073 \AA$

Cell parameters from 9630 reflections

$\theta=2.3-30.6^{\circ}$

$\mu=3.96 \mathrm{~mm}^{-1}$

$T=100 \mathrm{~K}$

Block, colourless

$0.44 \times 0.44 \times 0.12 \mathrm{~mm}$

5839 independent reflections

4658 reflections with $I>2 \sigma(I)$

$R_{\text {int }}=0.047$

$\theta_{\max }=30.6^{\circ}, \theta_{\min }=2.3^{\circ}$

$h=-16 \rightarrow 16$

$k=-19 \rightarrow 19$

$l=-17 \rightarrow 16$

Hydrogen site location: inferred from neighbouring sites

$\mathrm{H}$-atom parameters constrained

$w=1 /\left[\sigma^{2}\left(F_{\mathrm{o}}^{2}\right)+(0.0186 P)^{2}+1.9161 P\right]$

where $P=\left(F_{\mathrm{o}}^{2}+2 F_{\mathrm{c}}^{2}\right) / 3$

$(\Delta / \sigma)_{\max }=0.001$

$\Delta \rho_{\max }=1.25 \mathrm{e} \AA^{-3}$

$\Delta \rho_{\min }=-1.54 \mathrm{e} \AA^{-3}$

Extinction correction: SHELXL2018

(Sheldrick, 2015),

$\mathrm{Fc}^{*}=\mathrm{kFc}\left[1+0.001 \mathrm{xFc}^{2} \lambda^{3} / \sin (2 \theta)\right]^{-1 / 4}$

Extinction coefficient: 0.0120 (4) 


\section{Special details}

Geometry. All esds (except the esd in the dihedral angle between two 1.s. planes) are estimated using the full covariance matrix. The cell esds are taken into account individually in the estimation of esds in distances, angles and torsion angles; correlations between esds in cell parameters are only used when they are defined by crystal symmetry. An approximate (isotropic) treatment of cell esds is used for estimating esds involving l.s. planes.

Fractional atomic coordinates and isotropic or equivalent isotropic displacement parameters $\left(\hat{A}^{2}\right)$

\begin{tabular}{|c|c|c|c|c|}
\hline & $x$ & $y$ & $z$ & $U_{\text {iso }} * / U_{\text {eq }}$ \\
\hline Pt1 & 0.500000 & 0.500000 & 0.000000 & 0.01307 (4) \\
\hline $\mathrm{K} 1$ & 0.81107 (3) & $0.50085(2)$ & $0.38736(3)$ & $0.01684(7)$ \\
\hline O5 & $0.93998(11)$ & $0.65091(10)$ & $0.29546(12)$ & $0.0220(3)$ \\
\hline $\mathrm{O} 2$ & $0.71541(11)$ & $0.34705(10)$ & $0.50409(12)$ & $0.0232(3)$ \\
\hline $\mathrm{O} 4$ & $0.97580(12)$ & $0.45517(10)$ & $0.23439(12)$ & $0.0256(3)$ \\
\hline $\mathrm{O} 3$ & $0.82893(12)$ & $0.31294(10)$ & $0.30722(12)$ & $0.0254(3)$ \\
\hline $\mathrm{O} 1$ & $0.68912(13)$ & $0.54392(11)$ & $0.57027(12)$ & $0.0258(3)$ \\
\hline O6 & $0.84409(13)$ & $0.68057(10)$ & $0.50316(12)$ & $0.0273(3)$ \\
\hline $\mathrm{C} 2$ & $0.34516(17)$ & $0.47354(16)$ & $0.05042(17)$ & $0.0234(4)$ \\
\hline N2 & $0.25437(16)$ & $0.45768(17)$ & $0.07632(17)$ & $0.0358(4)$ \\
\hline N1 & $0.61178(17)$ & $0.50228(13)$ & $0.25023(17)$ & $0.0284(4)$ \\
\hline C9 & $0.99412(18)$ & $0.53486(16)$ & $0.15999(18)$ & $0.0268(4)$ \\
\hline H9A & 0.922679 & 0.549960 & 0.112661 & $0.032^{*}$ \\
\hline H9B & 1.054051 & 0.517845 & 0.109679 & $0.032^{*}$ \\
\hline $\mathrm{C} 1$ & $0.56960(16)$ & $0.50133(12)$ & $0.15887(17)$ & $0.0192(3)$ \\
\hline $\mathrm{C} 5$ & $0.68672(17)$ & $0.26882(14)$ & $0.42770(18)$ & $0.0265(4)$ \\
\hline $\mathrm{H} 5 \mathrm{~A}$ & 0.628172 & 0.290733 & 0.368065 & $0.032 *$ \\
\hline H5B & 0.654414 & 0.214014 & 0.468750 & $0.032 *$ \\
\hline $\mathrm{C} 10$ & $1.03093(17)$ & $0.62118(15)$ & $0.23170(19)$ & $0.0276(4)$ \\
\hline H10A & 1.098625 & 0.603787 & 0.283356 & $0.033^{*}$ \\
\hline H10B & 1.052471 & 0.675460 & 0.182981 & $0.033^{*}$ \\
\hline $\mathrm{C} 4$ & $0.61633(18)$ & $0.38299(16)$ & $0.5518(2)$ & $0.0309(4)$ \\
\hline $\mathrm{H} 4 \mathrm{~A}$ & 0.579757 & 0.329734 & 0.591811 & $0.037^{*}$ \\
\hline $\mathrm{H} 4 \mathrm{~B}$ & 0.560472 & 0.407414 & 0.491136 & $0.037 *$ \\
\hline C6 & $0.79142(17)$ & $0.23559(14)$ & $0.37517(17)$ & $0.0250(4)$ \\
\hline H6A & 0.852386 & 0.218411 & 0.434705 & $0.030^{*}$ \\
\hline H6B & 0.773614 & 0.177181 & 0.328031 & $0.030^{*}$ \\
\hline $\mathrm{C} 8$ & $0.9510(2)$ & $0.36800(15)$ & $0.1721(2)$ & $0.0335(5)$ \\
\hline $\mathrm{H} 8 \mathrm{~A}$ & 1.016517 & 0.350456 & 0.129004 & $0.040^{*}$ \\
\hline H8B & 0.882998 & 0.377767 & 0.117934 & $0.040^{*}$ \\
\hline $\mathrm{C} 14$ & $0.7268(2)$ & $0.62271(15)$ & $0.64242(18)$ & $0.0308(4)$ \\
\hline H14A & 0.795873 & 0.603522 & 0.691114 & $0.037^{*}$ \\
\hline H14B & 0.666168 & 0.640928 & 0.691447 & $0.037 *$ \\
\hline C11 & 0.97505 (19) & $0.72802(15)$ & $0.37123(18)$ & $0.0295(4)$ \\
\hline H11A & 1.002946 & 0.783790 & 0.328515 & $0.035^{*}$ \\
\hline H11B & 1.038243 & 0.705558 & 0.425670 & $0.035^{*}$ \\
\hline $\mathrm{C} 12$ & $0.8756(2)$ & $0.75891(14)$ & 0.43299 (19) & $0.0311(4)$ \\
\hline $\mathrm{H} 12 \mathrm{~A}$ & 0.896408 & 0.816886 & 0.479879 & $0.037^{*}$ \\
\hline H12B & 0.810247 & 0.776359 & 0.378360 & $0.037 *$ \\
\hline
\end{tabular}


supporting information

$\begin{array}{lllll}\text { C7 } & 0.9286(2) & 0.28768(15) & 0.2533(2) & 0.0329(5) \\ \text { H7A } & 0.916538 & 0.225258 & 0.212284 & 0.039^{*} \\ \text { H7B } & 0.994834 & 0.280173 & 0.310186 & 0.039^{*} \\ \text { C3 } & 0.65005(18) & 0.46399(16) & 0.63312(18) & 0.0280(4) \\ \text { H3A } & 0.583590 & 0.484034 & 0.673536 & 0.034^{*} \\ \text { H3B } & 0.711603 & 0.441769 & 0.689539 & 0.034^{*} \\ \text { C13 } & 0.7534(2) & 0.70708(15) & 0.5689(2) & 0.0342(5) \\ \text { H13A } & 0.684720 & 0.724656 & 0.518624 & 0.041^{*} \\ \text { H13B } & 0.775999 & 0.764336 & 0.616299 & 0.041^{*}\end{array}$

Atomic displacement parameters $\left(\AA^{2}\right)$

\begin{tabular}{lllllll}
\hline & $U^{11}$ & $U^{22}$ & $U^{33}$ & $U^{12}$ & $U^{13}$ & $U^{23}$ \\
\hline Pt1 & $0.01451(5)$ & $0.01192(5)$ & $0.01299(6)$ & $0.00149(3)$ & $0.00243(3)$ & $-0.00065(3)$ \\
K1 & $0.01690(16)$ & $0.01710(16)$ & $0.01701(17)$ & $-0.00121(12)$ & $0.00425(13)$ & $-0.00143(13)$ \\
O5 & $0.0216(6)$ & $0.0192(6)$ & $0.0254(7)$ & $-0.0033(5)$ & $0.0037(5)$ & $-0.0028(5)$ \\
O2 & $0.0223(6)$ & $0.0220(6)$ & $0.0260(7)$ & $-0.0027(5)$ & $0.0065(5)$ & $-0.0029(5)$ \\
O4 & $0.0296(7)$ & $0.0201(7)$ & $0.0283(7)$ & $0.0010(5)$ & $0.0104(6)$ & $-0.0033(6)$ \\
O3 & $0.0289(7)$ & $0.0185(6)$ & $0.0301(8)$ & $0.0011(5)$ & $0.0105(6)$ & $-0.0019(5)$ \\
O1 & $0.0319(7)$ & $0.0256(7)$ & $0.0213(7)$ & $-0.0017(6)$ & $0.0103(6)$ & $-0.0027(6)$ \\
O6 & $0.0382(8)$ & $0.0194(6)$ & $0.0255(7)$ & $-0.0025(6)$ & $0.0099(6)$ & $-0.0032(5)$ \\
C2 & $0.0251(9)$ & $0.0275(9)$ & $0.0176(8)$ & $0.0004(7)$ & $0.0018(7)$ & $-0.0031(7)$ \\
N2 & $0.0260(9)$ & $0.0547(13)$ & $0.0273(9)$ & $-0.0040(9)$ & $0.0062(7)$ & $-0.0055(9)$ \\
N1 & $0.0228(8)$ & $0.0430(11)$ & $0.0193(8)$ & $0.0027(7)$ & $0.0013(7)$ & $-0.0011(7)$ \\
C9 & $0.0278(10)$ & $0.0273(9)$ & $0.0272(10)$ & $0.0016(8)$ & $0.0138(8)$ & $0.0007(8)$ \\
C1 & $0.0156(8)$ & $0.0228(9)$ & $0.0193(8)$ & $0.0009(6)$ & $0.0030(6)$ & $-0.0009(6)$ \\
C5 & $0.0282(10)$ & $0.0226(9)$ & $0.0291(10)$ & $-0.0070(7)$ & $0.0052(8)$ & $-0.0030(8)$ \\
C10 & $0.0202(9)$ & $0.0268(9)$ & $0.0373(11)$ & $-0.0026(7)$ & $0.0101(8)$ & $0.0013(8)$ \\
C4 & $0.0261(10)$ & $0.0301(10)$ & $0.0390(12)$ & $-0.0041(8)$ & $0.0167(9)$ & $-0.0026(9)$ \\
C6 & $0.0300(10)$ & $0.0180(8)$ & $0.0268(10)$ & $-0.0017(7)$ & $0.0020(8)$ & $-0.0013(7)$ \\
C8 & $0.0426(12)$ & $0.0252(10)$ & $0.0355(12)$ & $-0.0025(9)$ & $0.0194(10)$ & $-0.0103(9)$ \\
C14 & $0.0422(12)$ & $0.0276(10)$ & $0.0243(10)$ & $0.0007(9)$ & $0.0131(9)$ & $-0.0070(8)$ \\
C11 & $0.0360(11)$ & $0.0226(9)$ & $0.0301(10)$ & $-0.0121(8)$ & $0.0037(8)$ & $-0.0037(8)$ \\
C12 & $0.0475(13)$ & $0.0174(9)$ & $0.0292(10)$ & $-0.0060(8)$ & $0.0075(9)$ & $-0.0046(8)$ \\
C7 & $0.0376(11)$ & $0.0207(9)$ & $0.0427(13)$ & $0.0024(8)$ & $0.0169(10)$ & $-0.0080(9)$ \\
C3 & $0.0280(10)$ & $0.0310(10)$ & $0.0273(10)$ & $0.0011(8)$ & $0.0162(8)$ & $0.0006(8)$ \\
C13 & $0.0496(13)$ & $0.0227(10)$ & $0.0322(11)$ & $0.0032(9)$ & $0.0147(10)$ & $-0.0070(8)$ \\
& & & & & & \\
\hline & & & & & &
\end{tabular}

Geometric parameters ( $\left.\AA,{ }^{\circ}\right)$

\begin{tabular}{llll}
\hline $\mathrm{Pt} 1-\mathrm{C} 2^{\mathrm{i}}$ & $1.996(2)$ & $\mathrm{C} 9-\mathrm{C} 10$ & $1.501(3)$ \\
$\mathrm{Pt} 1-\mathrm{C} 2$ & $1.996(2)$ & $\mathrm{C} 5-\mathrm{H} 5 \mathrm{~A}$ & 0.9900 \\
$\mathrm{Pt} 1-\mathrm{C} 1^{\mathrm{i}}$ & $1.991(2)$ & $\mathrm{C} 5-\mathrm{H} 5 \mathrm{~B}$ & 0.9900 \\
$\mathrm{Pt} 1-\mathrm{C} 1$ & $1.991(2)$ & $\mathrm{C} 5-\mathrm{C} 6$ & $1.497(3)$ \\
$\mathrm{K} 1-\mathrm{K} 1^{\mathrm{ii}}$ & $4.9761(9)$ & $\mathrm{C} 10-\mathrm{H} 10 \mathrm{~A}$ & 0.9900 \\
$\mathrm{~K} 1-\mathrm{O} 5$ & $2.8308(14)$ & $\mathrm{C} 10-\mathrm{H} 10 \mathrm{~B}$ & 0.9900 \\
$\mathrm{~K} 1-\mathrm{O} 2$ & $2.8133(14)$ & $\mathrm{C} 4-\mathrm{H} 4 \mathrm{~A}$ & 0.9900 \\
$\mathrm{~K} 1-\mathrm{O} 4$ & $2.8369(14)$ & $\mathrm{C} 4-\mathrm{H} 4 \mathrm{~B}$ & 0.9900
\end{tabular}




$$
\begin{aligned}
& \mathrm{K} 1-\mathrm{O} 3 \\
& \mathrm{~K} 1-\mathrm{O} 1 \\
& \mathrm{~K} 1-\mathrm{O} 6 \\
& \text { K1-N1 } \\
& \mathrm{K} 1-\mathrm{C} 4 \\
& \text { O5- } \mathrm{C} 10 \\
& \text { O5- } \mathrm{C} 11 \\
& \mathrm{O} 2-\mathrm{C} 5 \\
& \mathrm{O} 2-\mathrm{C} 4 \\
& \text { O4-C9 } \\
& \mathrm{O} 4-\mathrm{C} 8 \\
& \text { O3- } 6 \\
& \mathrm{O} 3-\mathrm{C} 7 \\
& \mathrm{O} 1-\mathrm{C} 14 \\
& \mathrm{O} 1-\mathrm{C} 3 \\
& \mathrm{O} 6-\mathrm{C} 12 \\
& \text { O6- } \mathrm{C} 13 \\
& \mathrm{C} 2-\mathrm{N} 2 \\
& \text { N1-C1 } \\
& \text { C9-H9A } \\
& \text { C9- } \mathrm{H} 9 \mathrm{~B} \\
& \mathrm{C} 22^{\mathrm{i}}-\mathrm{Pt} 1-\mathrm{C} 2 \\
& \mathrm{C} 1-\mathrm{Pt} 1-\mathrm{C} 2 \\
& \mathrm{C} 1^{\mathrm{i}}-\mathrm{Pt} 1-\mathrm{C}^{\mathrm{i}} \\
& \mathrm{C} 1-\mathrm{Pt} 1-\mathrm{C} 2^{\mathrm{i}} \\
& \mathrm{C} 1 \text { i }-\mathrm{Pt} 1-\mathrm{C} 2 \\
& \mathrm{C} 1-\mathrm{Pt} 1-\mathrm{C} 1 \\
& \text { O5-K1-K } 1^{\mathrm{ii}} \\
& \mathrm{O} 5-\mathrm{K} 1-\mathrm{O} 4 \\
& \text { O5-K1-O6 } \\
& \mathrm{O} 5-\mathrm{K} 1-\mathrm{C} 4 \\
& \mathrm{O} 2-\mathrm{K} 1-\mathrm{K} 1^{\mathrm{ii}} \\
& \mathrm{O} 2-\mathrm{K} 1-\mathrm{O} 5 \\
& \mathrm{O} 2-\mathrm{K} 1-\mathrm{O} 4 \\
& \mathrm{O} 2-\mathrm{K} 1-\mathrm{O} 6 \\
& \mathrm{O} 2-\mathrm{K} 1-\mathrm{C} 4 \\
& \mathrm{O} 4-\mathrm{K} 1-\mathrm{K} 1^{\mathrm{ii}} \\
& \mathrm{O} 4-\mathrm{K} 1-\mathrm{C} 4 \\
& \mathrm{O} 3-\mathrm{K} 1-\mathrm{K} 1^{i \mathrm{i}} \\
& \text { O3-K1-O5 } \\
& \mathrm{O} 3-\mathrm{K} 1-\mathrm{O} 2 \\
& \text { O3-K1-O4 } \\
& \text { O3-K1-O1 } \\
& \text { O3-K1-O6 } \\
& \mathrm{O} 3-\mathrm{K} 1-\mathrm{C} 4 \\
& \mathrm{O} 1-\mathrm{K} 1-\mathrm{K} 1^{\mathrm{ii}} \\
& \mathrm{O} 1-\mathrm{K} 1-\mathrm{O} 5
\end{aligned}
$$

$2.7642(14)$

$2.7691(14)$

2.8354 (14)

$2.732(2)$

3.527 (2)

$1.422(2)$

$1.427(2)$

$1.428(2)$

$1.425(2)$

$1.435(3)$

$1.424(2)$

$1.427(2)$

$1.425(2)$

$1.426(2)$

$1.426(3)$

$1.429(2)$

$1.421(3)$

$1.155(3)$

$1.154(3)$

0.9900

0.9900

180.0

$91.47(8)$

$91.47(8)$

$88.53(8)$

$88.53(8)$

180.0

74.34 (3)

$59.78(4)$

$59.94(4)$

$160.31(5)$

96.09 (3)

$170.43(4)$

$118.28(4)$

$117.20(4)$

$22.59(4)$

$73.68(3)$

139.85 (5)

$95.19(3)$

119.15 (4)

$61.00(4)$

59.77 (4)

121.99 (4)

$165.50(5)$

$80.51(5)$

$94.33(3)$

118.53 (4)
$\mathrm{C} 4-\mathrm{C} 3$

C6-H6A

C6-H6B

$\mathrm{C} 8-\mathrm{H} 8 \mathrm{~A}$

$\mathrm{C} 8-\mathrm{H} 8 \mathrm{~B}$

$\mathrm{C} 8-\mathrm{C} 7$

C14-H14A

C14-H14B

C14-C13

C11-H11A

C11-H11B

$\mathrm{C} 11-\mathrm{C} 12$

C12-H12A

C12-H12B

C7-H7A

C7-H7B

$\mathrm{C} 3-\mathrm{H} 3 \mathrm{~A}$

$\mathrm{C} 3-\mathrm{H} 3 \mathrm{~B}$

C13-H13A

C13-H13B

$\mathrm{O} 2-\mathrm{C} 5-\mathrm{H} 5 \mathrm{~B}$

$\mathrm{O} 2-\mathrm{C} 5-\mathrm{C} 6$

$\mathrm{H} 5 \mathrm{~A}-\mathrm{C} 5-\mathrm{H} 5 \mathrm{~B}$

C6- C5-H5A

C6- C5-H5B

$\mathrm{O} 5-\mathrm{C} 10-\mathrm{C} 9$

$\mathrm{O} 5-\mathrm{C} 10-\mathrm{H} 10 \mathrm{~A}$

$\mathrm{O} 5-\mathrm{C} 10-\mathrm{H} 10 \mathrm{~B}$

C9- $\mathrm{C} 10-\mathrm{H} 10 \mathrm{~A}$

C9- $\mathrm{C} 10-\mathrm{H} 10 \mathrm{~B}$

$\mathrm{H} 10 \mathrm{~A}-\mathrm{C} 10-\mathrm{H} 10 \mathrm{~B}$

$\mathrm{K} 1-\mathrm{C} 4-\mathrm{H} 4 \mathrm{~A}$

$\mathrm{K} 1-\mathrm{C} 4-\mathrm{H} 4 \mathrm{~B}$

$\mathrm{O} 2-\mathrm{C} 4-\mathrm{K} 1$

$\mathrm{O} 2-\mathrm{C} 4-\mathrm{H} 4 \mathrm{~A}$

$\mathrm{O} 2-\mathrm{C} 4-\mathrm{H} 4 \mathrm{~B}$

$\mathrm{O} 2-\mathrm{C} 4-\mathrm{C} 3$

$\mathrm{H} 4 \mathrm{~A}-\mathrm{C} 4-\mathrm{H} 4 \mathrm{~B}$

$\mathrm{C} 3-\mathrm{C} 4-\mathrm{K} 1$

$\mathrm{C} 3-\mathrm{C} 4-\mathrm{H} 4 \mathrm{~A}$

$\mathrm{C} 3-\mathrm{C} 4-\mathrm{H} 4 \mathrm{~B}$

$\mathrm{O} 3-\mathrm{C} 6-\mathrm{C} 5$

$\mathrm{O} 3-\mathrm{C} 6-\mathrm{H} 6 \mathrm{~A}$

$\mathrm{O} 3-\mathrm{C} 6-\mathrm{H} 6 \mathrm{~B}$

$\mathrm{C} 5-\mathrm{C} 6-\mathrm{H} 6 \mathrm{~A}$

$\mathrm{C} 5-\mathrm{C} 6-\mathrm{H} 6 \mathrm{~B}$
$1.503(3)$

0.9900

0.9900

0.9900

0.9900

$1.503(3)$

0.9900

0.9900

$1.500(3)$

0.9900

0.9900

1.493 (3)

0.9900

0.9900

0.9900

0.9900

0.9900

0.9900

0.9900

0.9900

109.7

109.75 (16)

108.2

109.7

109.7

109.71 (16)

109.7

109.7

109.7

109.7

108.2

159.0

82.0

49.29 (9)

109.8

109.8

109.49 (17)

108.2

82.54 (11)

109.8

109.8

108.32 (16)

110.0

110.0

110.0

110.0 


\begin{tabular}{|c|c|c|c|}
\hline $\mathrm{O} 1-\mathrm{K} 1-\mathrm{O} 2$ & $61.13(4)$ & $\mathrm{H} 6 \mathrm{~A}-\mathrm{C} 6-\mathrm{H} 6 \mathrm{~B}$ & 108.4 \\
\hline $\mathrm{O} 1-\mathrm{K} 1-\mathrm{O} 4$ & $167.99(5)$ & $\mathrm{O} 4-\mathrm{C} 8-\mathrm{H} 8 \mathrm{~A}$ & 109.9 \\
\hline $\mathrm{O} 1-\mathrm{K} 1-\mathrm{O} 6$ & $59.40(4)$ & $\mathrm{O} 4-\mathrm{C} 8-\mathrm{H} 8 \mathrm{~B}$ & 109.9 \\
\hline $\mathrm{O} 1-\mathrm{K} 1-\mathrm{C} 4$ & $42.15(5)$ & $\mathrm{O} 4-\mathrm{C} 8-\mathrm{C} 7$ & $108.77(18)$ \\
\hline $\mathrm{O} 6-\mathrm{K} 1-\mathrm{K} 1^{\mathrm{ii}}$ & $70.44(3)$ & $\mathrm{H} 8 \mathrm{~A}-\mathrm{C} 8-\mathrm{H} 8 \mathrm{~B}$ & 108.3 \\
\hline $\mathrm{O} 6-\mathrm{K} 1-\mathrm{O} 4$ & $115.57(4)$ & $\mathrm{C} 7-\mathrm{C} 8-\mathrm{H} 8 \mathrm{~A}$ & 109.9 \\
\hline $\mathrm{O} 6-\mathrm{K} 1-\mathrm{C} 4$ & $101.40(5)$ & $\mathrm{C} 7-\mathrm{C} 8-\mathrm{H} 8 \mathrm{~B}$ & 109.9 \\
\hline $\mathrm{N} 1-\mathrm{K} 1-\mathrm{K} 1^{\mathrm{ii}}$ & $175.95(5)$ & $\mathrm{O} 1-\mathrm{C} 14-\mathrm{H} 14 \mathrm{~A}$ & 110.2 \\
\hline $\mathrm{N} 1-\mathrm{K} 1-\mathrm{O} 5$ & $102.88(5)$ & $\mathrm{O} 1-\mathrm{C} 14-\mathrm{H} 14 \mathrm{~B}$ & 110.2 \\
\hline $\mathrm{N} 1-\mathrm{K} 1-\mathrm{O} 2$ & $86.68(5)$ & $\mathrm{O} 1-\mathrm{C} 14-\mathrm{C} 13$ & $107.71(17)$ \\
\hline $\mathrm{N} 1-\mathrm{K} 1-\mathrm{O} 4$ & $102.40(5)$ & $\mathrm{H} 14 \mathrm{~A}-\mathrm{C} 14-\mathrm{H} 14 \mathrm{~B}$ & 108.5 \\
\hline $\mathrm{N} 1-\mathrm{K} 1-\mathrm{O} 3$ & $83.55(5)$ & $\mathrm{C} 13-\mathrm{C} 14-\mathrm{H} 14 \mathrm{~A}$ & 110.2 \\
\hline $\mathrm{N} 1-\mathrm{K} 1-\mathrm{O} 1$ & $89.59(5)$ & $\mathrm{C} 13-\mathrm{C} 14-\mathrm{H} 14 \mathrm{~B}$ & 110.2 \\
\hline $\mathrm{N} 1-\mathrm{K} 1-\mathrm{O} 6$ & $110.92(5)$ & $\mathrm{O} 5-\mathrm{C} 11-\mathrm{H} 11 \mathrm{~A}$ & 109.9 \\
\hline $\mathrm{N} 1-\mathrm{K} 1-\mathrm{C} 4$ & $76.81(6)$ & $\mathrm{O} 5-\mathrm{C} 11-\mathrm{H} 11 \mathrm{~B}$ & 109.9 \\
\hline $\mathrm{C} 4-\mathrm{K} 1-\mathrm{K} 1^{\mathrm{ii}}$ & $106.82(4)$ & $\mathrm{O} 5-\mathrm{C} 11-\mathrm{C} 12$ & $109.03(17)$ \\
\hline $\mathrm{C} 10-\mathrm{O} 5-\mathrm{K} 1$ & $116.61(11)$ & $\mathrm{H} 11 \mathrm{~A}-\mathrm{C} 11-\mathrm{H} 11 \mathrm{~B}$ & 108.3 \\
\hline $\mathrm{C} 10-\mathrm{O} 5-\mathrm{C} 11$ & $111.15(15)$ & $\mathrm{C} 12-\mathrm{C} 11-\mathrm{H} 11 \mathrm{~A}$ & 109.9 \\
\hline $\mathrm{C} 11-\mathrm{O} 5-\mathrm{K} 1$ & $115.51(11)$ & $\mathrm{C} 12-\mathrm{C} 11-\mathrm{H} 11 \mathrm{~B}$ & 109.9 \\
\hline $\mathrm{C} 5-\mathrm{O} 2-\mathrm{K} 1$ & $109.45(11)$ & $\mathrm{O} 6-\mathrm{C} 12-\mathrm{C} 11$ & $109.03(17)$ \\
\hline $\mathrm{C} 4-\mathrm{O} 2-\mathrm{K} 1$ & $108.12(11)$ & $\mathrm{O} 6-\mathrm{C} 12-\mathrm{H} 12 \mathrm{~A}$ & 109.9 \\
\hline $\mathrm{C} 4-\mathrm{O} 2-\mathrm{C} 5$ & $110.99(15)$ & $\mathrm{O} 6-\mathrm{C} 12-\mathrm{H} 12 \mathrm{~B}$ & 109.9 \\
\hline $\mathrm{C} 9-\mathrm{O} 4-\mathrm{K} 1$ & $111.94(11)$ & $\mathrm{C} 11-\mathrm{C} 12-\mathrm{H} 12 \mathrm{~A}$ & 109.9 \\
\hline $\mathrm{C} 8-\mathrm{O} 4-\mathrm{K} 1$ & $113.54(11)$ & $\mathrm{C} 11-\mathrm{C} 12-\mathrm{H} 12 \mathrm{~B}$ & 109.9 \\
\hline $\mathrm{C} 8-\mathrm{O} 4-\mathrm{C} 9$ & $110.80(17)$ & $\mathrm{H} 12 \mathrm{~A}-\mathrm{C} 12-\mathrm{H} 12 \mathrm{~B}$ & 108.3 \\
\hline $\mathrm{C} 6-\mathrm{O} 3-\mathrm{K} 1$ & $117.58(11)$ & $\mathrm{O} 3-\mathrm{C} 7-\mathrm{C} 8$ & $107.85(17)$ \\
\hline $\mathrm{C} 7-\mathrm{O} 3-\mathrm{K} 1$ & $118.16(11)$ & $\mathrm{O} 3-\mathrm{C} 7-\mathrm{H} 7 \mathrm{~A}$ & 110.1 \\
\hline $\mathrm{C} 7-\mathrm{O} 3-\mathrm{C} 6$ & $112.30(15)$ & $\mathrm{O} 3-\mathrm{C} 7-\mathrm{H} 7 \mathrm{~B}$ & 110.1 \\
\hline $\mathrm{C} 14-\mathrm{O} 1-\mathrm{K} 1$ & $118.68(12)$ & $\mathrm{C} 8-\mathrm{C} 7-\mathrm{H} 7 \mathrm{~A}$ & 110.1 \\
\hline $\mathrm{C} 3-\mathrm{O} 1-\mathrm{K} 1$ & $117.32(11)$ & $\mathrm{C} 8-\mathrm{C} 7-\mathrm{H} 7 \mathrm{~B}$ & 110.1 \\
\hline $\mathrm{C} 3-\mathrm{O} 1-\mathrm{C} 14$ & $111.44(16)$ & $\mathrm{H} 7 \mathrm{~A}-\mathrm{C} 7-\mathrm{H} 7 \mathrm{~B}$ & 108.4 \\
\hline $\mathrm{C} 12-\mathrm{O} 6-\mathrm{K} 1$ & $113.75(11)$ & $\mathrm{O} 1-\mathrm{C} 3-\mathrm{C} 4$ & $108.13(17)$ \\
\hline $\mathrm{C} 13-\mathrm{O} 6-\mathrm{K} 1$ & $114.36(12)$ & $\mathrm{O} 1-\mathrm{C} 3-\mathrm{H} 3 \mathrm{~A}$ & 110.1 \\
\hline $\mathrm{C} 13-\mathrm{O} 6-\mathrm{C} 12$ & $111.85(16)$ & $\mathrm{O} 1-\mathrm{C} 3-\mathrm{H} 3 \mathrm{~B}$ & 110.1 \\
\hline $\mathrm{N} 2-\mathrm{C} 2-\mathrm{Pt} 1$ & $177.97(18)$ & $\mathrm{C} 4-\mathrm{C} 3-\mathrm{H} 3 \mathrm{~A}$ & 110.1 \\
\hline $\mathrm{C} 1-\mathrm{N} 1-\mathrm{K} 1$ & $146.76(17)$ & $\mathrm{C} 4-\mathrm{C} 3-\mathrm{H} 3 \mathrm{~B}$ & 110.1 \\
\hline $\mathrm{O} 4-\mathrm{C} 9-\mathrm{H} 9 \mathrm{~A}$ & 110.2 & $\mathrm{H} 3 \mathrm{~A}-\mathrm{C} 3-\mathrm{H} 3 \mathrm{~B}$ & 108.4 \\
\hline $\mathrm{O} 4-\mathrm{C} 9-\mathrm{H} 9 \mathrm{~B}$ & 110.2 & $\mathrm{O} 6-\mathrm{C} 13-\mathrm{C} 14$ & $109.05(17)$ \\
\hline $\mathrm{O} 4-\mathrm{C} 9-\mathrm{C} 10$ & $107.64(17)$ & $\mathrm{O} 6-\mathrm{C} 13-\mathrm{H} 13 \mathrm{~A}$ & 109.9 \\
\hline $\mathrm{H} 9 \mathrm{~A}-\mathrm{C} 9-\mathrm{H} 9 \mathrm{~B}$ & 108.5 & $\mathrm{O} 6-\mathrm{C} 13-\mathrm{H} 13 \mathrm{~B}$ & 109.9 \\
\hline $\mathrm{C} 10-\mathrm{C} 9-\mathrm{H} 9 \mathrm{~A}$ & 110.2 & $\mathrm{C} 14-\mathrm{C} 13-\mathrm{H} 13 \mathrm{~A}$ & 109.9 \\
\hline $\mathrm{C} 10-\mathrm{C} 9-\mathrm{H} 9 \mathrm{~B}$ & 110.2 & $\mathrm{C} 14-\mathrm{C} 13-\mathrm{H} 13 \mathrm{~B}$ & 109.9 \\
\hline $\mathrm{N} 1-\mathrm{C} 1-\mathrm{Pt} 1$ & $178.81(18)$ & $\mathrm{H} 13 \mathrm{~A}-\mathrm{C} 13-\mathrm{H} 13 \mathrm{~B}$ & 108.3 \\
\hline $\mathrm{O} 2-\mathrm{C} 5-\mathrm{H} 5 \mathrm{~A}$ & 109.7 & & \\
\hline
\end{tabular}

Symmetry codes: (i) $-x+1,-y+1,-z$; (ii) $-x+2,-y+1,-z+1$. 
supporting information

Hydrogen-bond geometry $\left(\AA,{ }^{\circ}\right)$

\begin{tabular}{lllll}
\hline$D-\mathrm{H} \cdots A$ & $D-\mathrm{H}$ & $\mathrm{H} \cdots A$ & $D \cdots A$ & $D-\mathrm{H} \cdots A$ \\
\hline $\mathrm{C} 3-\mathrm{H} 3 A \cdots \mathrm{N} 1^{\mathrm{iii}}$ & 0.99 & 2.54 & $3.510(3)$ & 165 \\
$\mathrm{C} 9-\mathrm{H} 9 B \cdots \mathrm{N} 2^{\mathrm{iv}}$ & 0.99 & 2.55 & $3.459(3)$ & 152 \\
\hline
\end{tabular}

Symmetry codes: (iii) $-x+1,-y+1,-z+1$; (iv) $x+1, y, z$. 